\title{
Electromagnetic and mechanical control of slip: laboratory experiments with slider system
}

\author{
T. Chelidze ${ }^{1}$ and O. Lursmanashvili ${ }^{2}$ \\ ${ }^{1}$ Institute of Geophysics, Georgian Academy of Sciences, 1 Alexidze str, 380093 Tbilisi, Georgia \\ 2 Tbilisi State University, 1, Chavchavadze Ave, 380028, Tbilisi, Georgia
}

Received: 3 December 2002 - Revised: 9 June 2003 - Accepted: 12 July 2003

\begin{abstract}
Field and laboratory data reveal the possibility of a significant coupling of elastic and electromagnetic (EM) fields that affect (hamper or initiate) slip. In this work we try to prove experimentally the possibility of controlling the slip regime by relatively weak mechanical or EM impact, in the way it has been done in nonlinear dynamic experiments on the control of chaos. The experimental setup consisted of a system of two plates of roughly finished basalt, where a constant pulling force was applied to the upper (sliding) plate. In addition, the same plate was subjected to mechanical or electric periodic perturbations, which are much weaker when compared to the pulling force. Quite different regimes of slip were excited depending on the amplitude and the frequency of applied weak perturbations. The observed regimes of slip vary from perfect synchronization of slip events, recorded as acoustic emission bursts with the perturbing periodic mechanical or EM impact, to their complete desynchronization. We consider the obtained results as evidence that it is possible to control slip by the application of weak periodic perturbations. The phenomenon can be explained in terms of nonlinear dynamics and synchronization theory.
\end{abstract}

\section{Introduction}

It has been claimed recently (Tarasov et al., 1999) that strong electromagnetic (EM) discharges using both magnetohydrodynamic (MHD) and "cold" sources, realized by the Moscow Institute of High Temperatures (IVTAN) at Bishkek's test area (Central Asia), can change the seismic regime in the region. The nonlinear analysis of the inter-event (waiting) time sequences in the regional seismological catalogue of the Bishkek area, carried out by several qualitative and quantitative methods, reveals the clear evidence of changing the seismic regime of the region during the period of EM discharge experiments at least in the timing of seismic events: the waiting times of earthquakes are much more regular in

Correspondence to: T. Chelidze (chelidze@ig.acnet.ge) this period compared to the periods before and after the tests (Chelidze and Matcharashvili, 2003). The laboratory experiments, where strong EM pulses were applied to the mechanical system driven close to the critical state, show that the EM impact can either initiate or hamper instability (here, slip) occurrence, depending on the mutual orientation of the slip surface and electrical field (Chelidze et al., 2002).

All of these observations imply that the EM field can significantly affect and even control the mechanical stability of systems that are close to the critical state. Therefore, it seems important to confirm by laboratory experiments the possibility of EM control of mechanical behavior of systems that mimic large-scale fault dynamics, namely the systems manifesting a stick-slip effect. The present paper deals with the results of experiments with the spring-slider system subjected to a constant pull, with weak mechanical or EM periodic forces superimposed on it.

\section{Experimental setup}

The mechanical part of the experimental system consisted of supporting (fixed) and sliding plates of roughly finished basalt samples; the average height of surface asperities was $0.1-0.2 \mathrm{~mm}$. The sliding block was driven with a constant velocity $V_{d}$ by a motor with reducers. It was possible to change the drag velocity from 0.5 to $7 \mathrm{~mm} / \mathrm{s}$. A dynamometer was attached to the sliding sample. The stiffness of pulling spring $K_{S}$ was either $150 \mathrm{~N} / \mathrm{m}$ or $1000 \mathrm{~N} / \mathrm{m}$. The pulling force $F_{p}$ varied from 3.5 to $15.0 \mathrm{~N}$. The coefficient of friction for basalt, measured by inclined plane tests, was 0.47 (Chelidze et al., 2002). The static friction resistance force was in the range of $5-15 \mathrm{~N}$ and in dynamic conditions the force was $<1 \mathrm{~N}$.

The acoustic emissions (AE) accompanying the elementary slip events were recorded on a PC sound card. The sensor for the AE was lead circonate-titanate with a natural frequency of $100 \mathrm{KHz}$. 


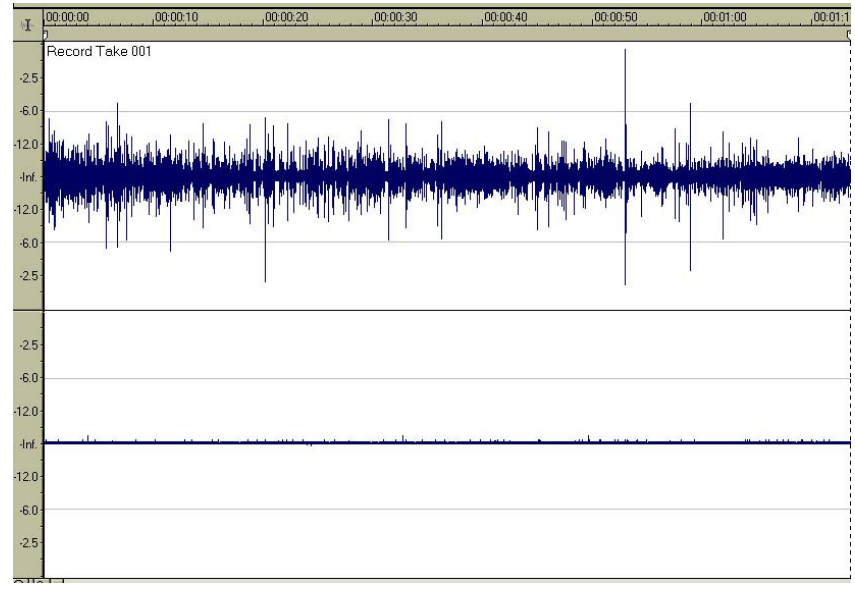

(a)

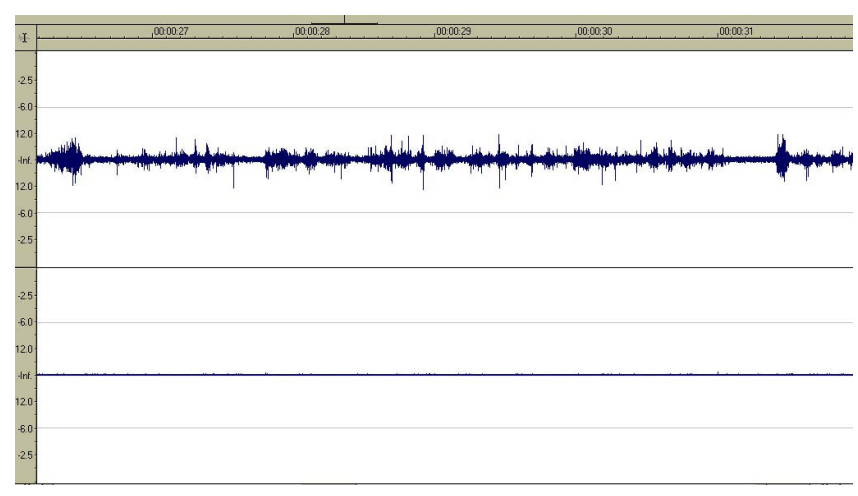

(b)

Fig. 1. Acoustic emission during slip without periodical impact: (a) the whole recording; (b) part of the recording with an expanded time axis. Values were for a stiff spring $\left(K_{S}=1000 \mathrm{~N}\right)$, pulling force $F_{p}=3.5 \mathrm{~N}$, and normal (nominal) stress $\sigma_{n}=2 \mathrm{kPa}$. The mean velocity of drag $V_{d}$ in this and the following experiments was $0.25 \pm 0.05 \mathrm{~cm} / \mathrm{s}$. The $y$-axis on all the figures shows the amplitude of signal in $\mathrm{dB}$. The upper trace shows the acoustic emission during stick-slip; the lower trace records the superimposed periodic excitation, which in this case is absent. On the time axis the first two positions correspond to hours, the next two to minutes and the last numbers are for seconds and milliseconds from the beginning of the experiment. All experiments were performed on basalt samples.

Weak periodic mechanical or electrical perturbations were superimposed on the system, in order to find the conditions that allow for control of the slip. For electrical impact the standard sound generator and a system of transformers were used (frequencies varied from 25 to $100 \mathrm{~Hz}$ and the voltage from 100 to $1100 \mathrm{~V}$ ). The electrical field, applied by foil electrodes glued to the external facets of samples, was either normal to the slip surface or parallel to it.

The superimposed periodic mechanical oscillations were

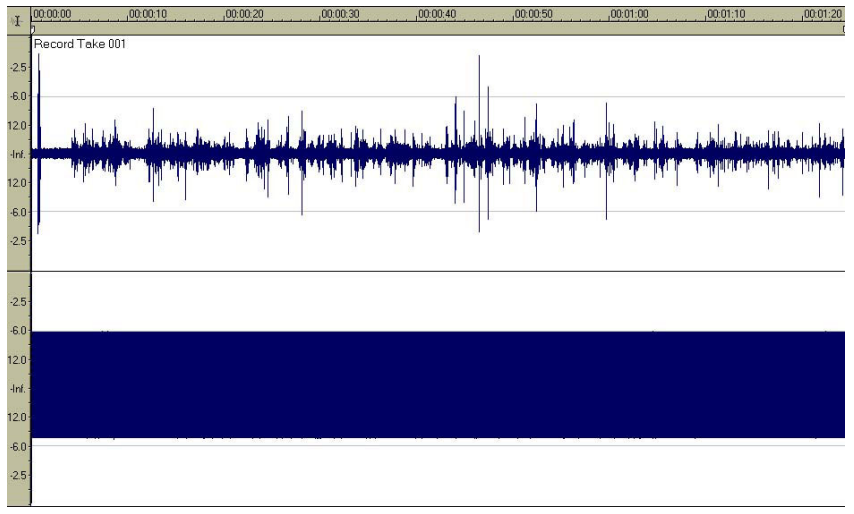

(a)

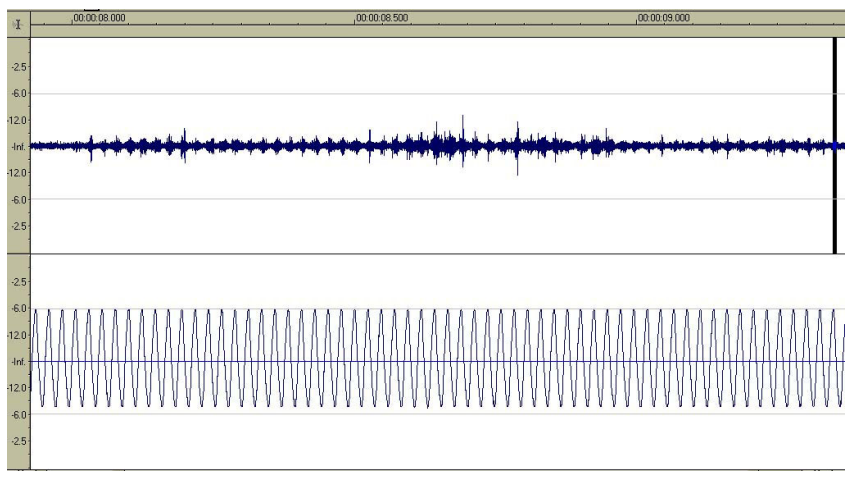

(b)

Fig. 2. Control of slip process by the weak periodic mechanical impact: (a) the whole recording and (b) part of the recording with the synchronization interval expanded. The upper trace is the acoustic emission during slip and the lower trace the periodic impact, $f=50 \mathrm{~Hz}$. Values were for a stiff spring $\left(K_{s}=1000 \mathrm{~N}\right)$, pulling force $F_{p}=8.5 \mathrm{~N}$, and normal (nominal) stress $\sigma_{n}=2 \mathrm{kPa}$. The weak perturbation was applied simultaneously with the pull. Synchronization appears $4.2 \mathrm{~s}$ after the beginning of the experiment. Synchronization periods last 5-10s and alternate with nonsynchronized intervals.

generated by a periodic voltage applied to the magnetoelectric system, a small size seismograph sensor which was attached to the front of the sliding sample. The maximal amplitude of oscillation of the sensor's inertial mass was around $2 \mathrm{~mm}$ and the natural frequency $20 \mathrm{~Hz}$. The periodic mechanical force was directed parallel to the slip surface, with its amplitude on the order of $0.1 \mathrm{~N}$ at $20 \mathrm{~Hz}(0.5 \mathrm{~N}$ at $50 \mathrm{~Hz})$, much less than the dragging force $(5-15 \mathrm{~N})$. The temperature during the experiments was $25 \pm 3^{\circ} \mathrm{C}$ and the atmospheric humidity $60 \pm 10 \%$. 


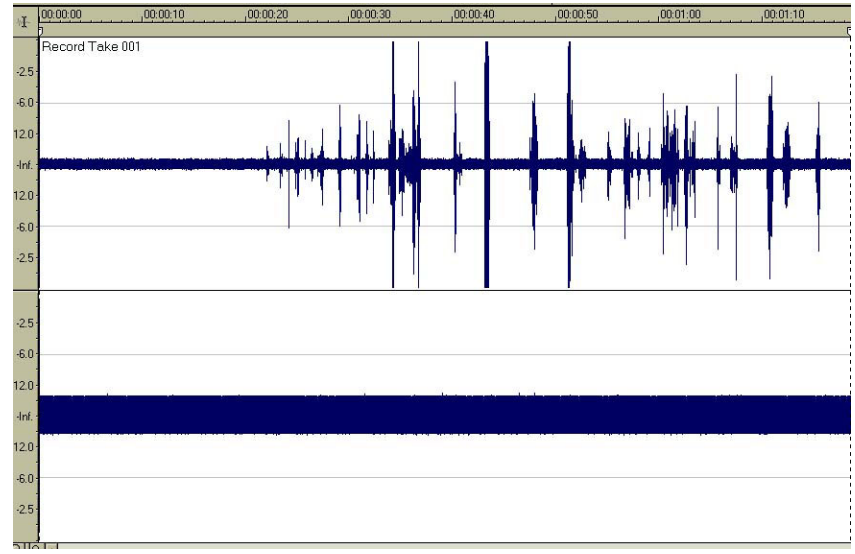

(a)

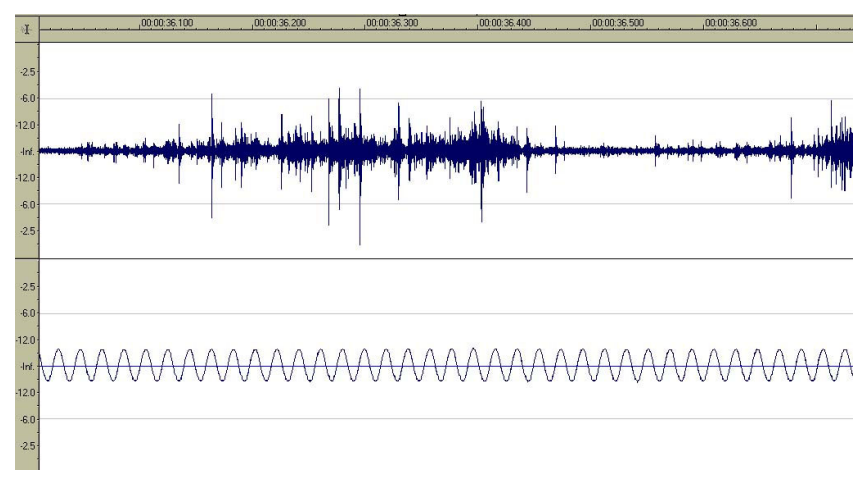

(b)

Fig. 3. Slip process with the superimposed EM periodic impact, $V_{a}=300 \mathrm{~V}, f=80 \mathrm{~Hz}, K_{s}=150 \mathrm{~N}, F_{p}=8.5 \mathrm{~N}, \sigma_{n}=3 \mathrm{kPa}$.

(a) The whole recording and (b) part of the recording expanded. The synchronization effect is absent at this frequency. In this and following experiments the EM excitation was applied simultaneously with a pull.

\section{Experimental results}

In our experiments the following parameters were varied:

(i) The stiffness of the spring, $K_{s}$;

(ii) The frequency, $f$, of the superimposed periodical perturbation;

(iii) The amplitude of the excitation (applied voltage $V_{a}$ );

(iv) The direction of the applied electrical field; $(v)$ the velocity of drag, $V_{d} ;\left(v_{i}\right)$ the normal (nominal) stress $\sigma_{n}$.

Seven cases were studied, and are described in each of the following sections.

- Case 1: Pulling of the sliding sample without any additional impact. These experiments were done in order to study the background behavior of the system during

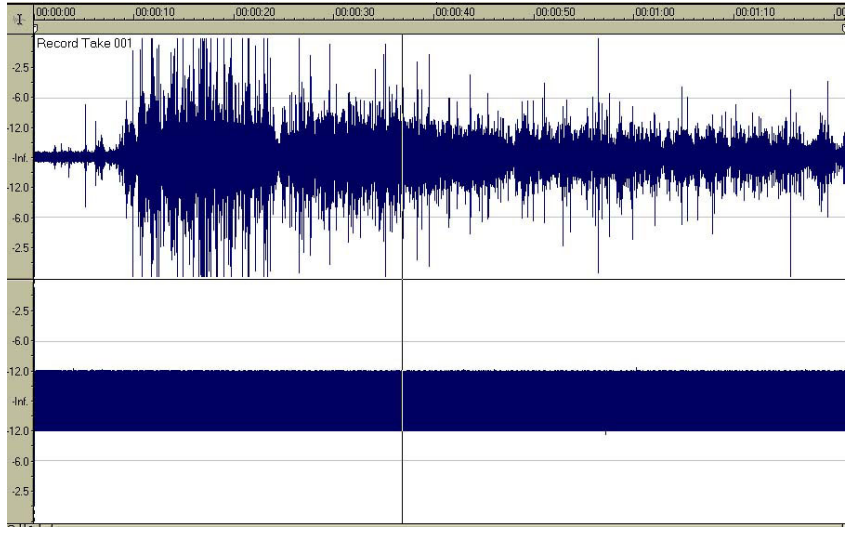

(a)

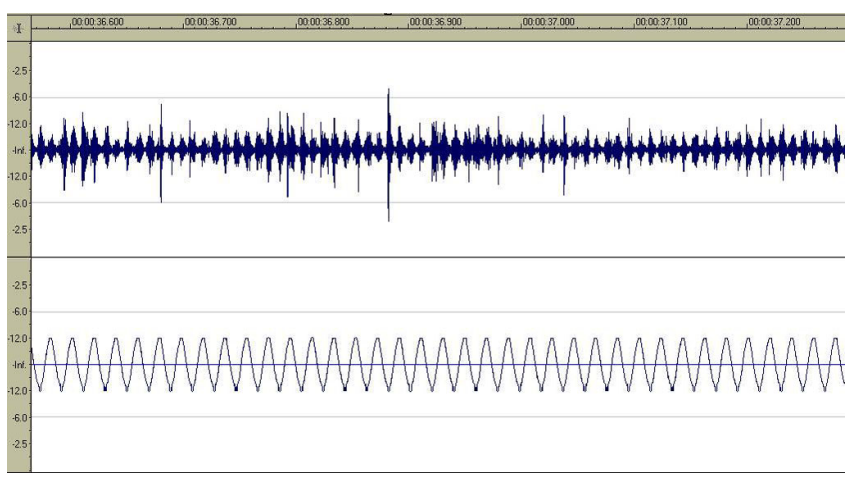

(b)

Fig. 4. Control of slip by the EM periodic impact. $V_{a}=500 \mathrm{~V}$, $f=80 \mathrm{~Hz}, K_{s}=150 \mathrm{~N}, F_{p}=8.5 \mathrm{~N}, \sigma_{n}=3 \mathrm{kPa}$. (a) The whole recording and (b) part of the recording expanded. The synchronization effect appears $8.5 \mathrm{~s}$ after the beginning of the experiment.

the conventional stick-slip. The results are presented in Fig. 1a,b. Here and in other figures, the $x$-axis corresponds to time and the $y$-axis to the amplitude of the signal in $\mathrm{dB}$. It is evident that under these conditions $\mathrm{AE}$ events do not manifest any visible periodicity at the time scale of several milliseconds.

- Case 2: Applying EM and mechanical periodical perturbations to the system of supporting and upper samples in static conditions (no pulling). The experiments were made in order to assess the background noises.

- Case 3: The slip process with an additional weak (0.1$0.5 \mathrm{~N})$ mechanical periodic force. A typical case is shown in Figs. 2a, b. The upper trace corresponds to AE signals generated by elementary slip events and the lower one to the superimposed periodic mechanical perturbation. On the large (compressed) time scale (Fig. 2a, upper trace) it is impossible to see any periodicity in the AE recording. However, after stretching the time axis, 


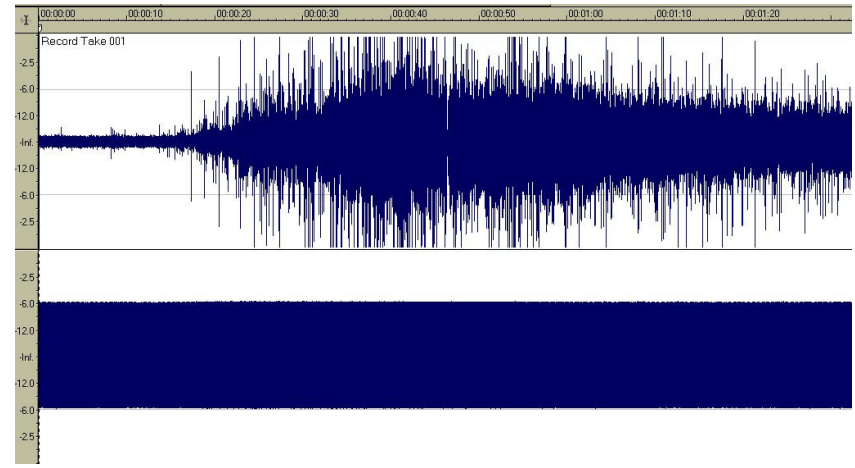

(a)

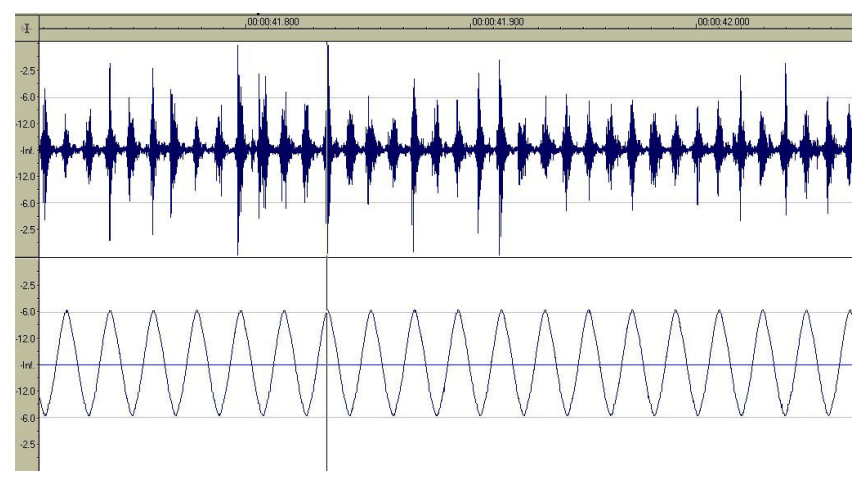

(c)

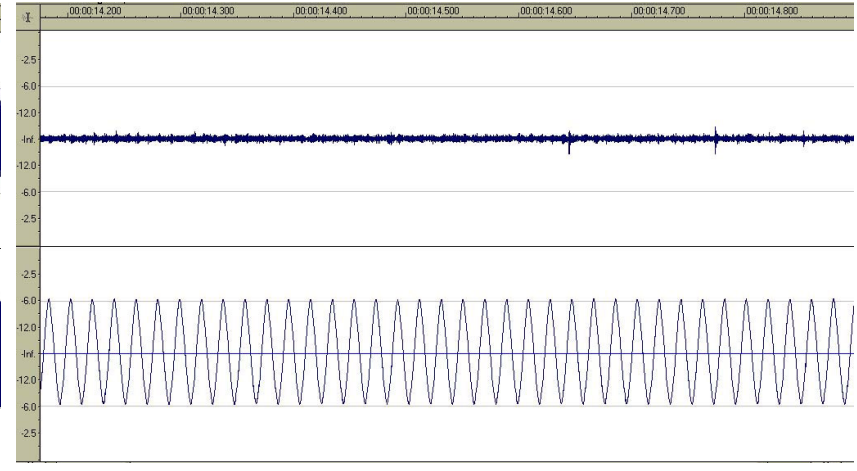

(b)

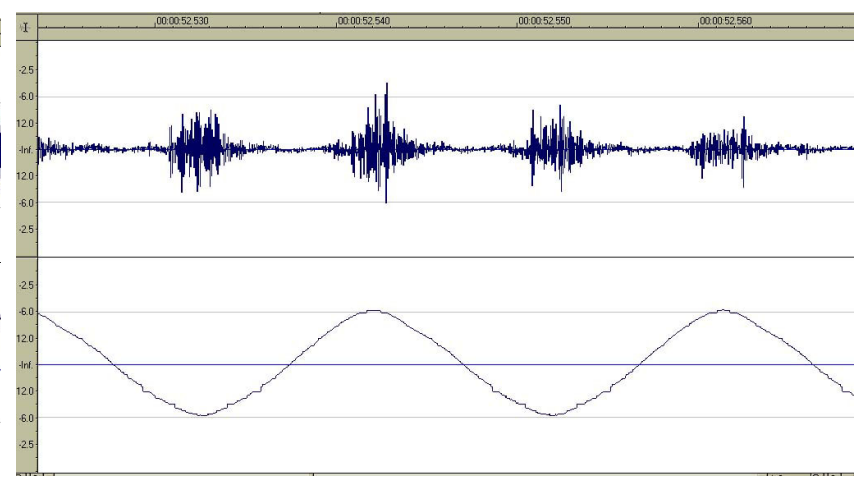

(d)

Fig. 5. Control of slip by EM periodic impact. $V_{a}=1000 \mathrm{~V}, f=80 \mathrm{~Hz}, K_{s}=150 \mathrm{~N}, F_{p}=8.5 \mathrm{~N}, \sigma_{n}=3 \mathrm{kPa}$. (a) The whole recording; (b) part of the recording with the onset of weak synchronization expanded; (c) part of the recording with well developed synchronization expanded; (d) the same part at the larger expansion of the time axis. The dominant frequency of oscillations during the acoustic burst is $12 \mathrm{kHz}$. The evolution of the synchronization process was as follows: weak synchronization, like in Fig. 5b, appears immediately after the application of the periodic impact, then pronounced synchronization begins after $18.2 \mathrm{~s}$ and finally, a very strong one, like in Fig. 5c begins after $22 \mathrm{~s}$ and lasts until the end of the slip.

we see that the periodic perturbation imposes on the slip a clear periodicity within slip-generated AE packages: acoustic events, generated by elementary slip events, begin at the definite phase of the periodic impact, i.e. soon after passing the zero value at the increasing amplitude of sinusoid, that is once per period. The maxima of the AE packages coincide exactly with the maxima of the perturbing force (Fig. 2b). In some cases, only partial synchronization was observed with only part of the maxima on the AE channel coinciding with the particular phase of the superimposed periodic impact.

- Case 4: Slip with a superimposed periodic EM field, oriented normally to the slip surface. Figures 3-5 show the response of slip to the low-frequency EM perturbation $(f=80 \mathrm{~Hz})$ at applied voltages $V_{a}$, respectively, 300,500 and $1050 \mathrm{~V}$. It is evident that the significant synchronization at this frequency occurs at $V_{a}=500 \mathrm{~V}$. The minimal voltage, under which the synchronization was still observable in some parts of the recording, is $300 \mathrm{~V}$ at a frequency of $40 \mathrm{~Hz}$. At smaller voltages slip is not correlated with the excitation phase. The phases of correlation under EM excitation differ from the regularity observed under mechanical impact: in the former case, the AE events (microslips) occur twice per period, soon after the beginning of both decreasing and increasing phases of oscillation. The maxima of $\mathrm{AE}$ coincide exactly with extreme points of oscillation. At higher frequencies the synchronization effect is less pronounced.

- Case 5: Synchronization was observed only at some definite values of the set of parameters $\left(V_{d}, K_{s}, f, V_{a}\right)$. These values are presented in the figure captions. Our goal was to prove the possibility of a nonlinear control of slip. A compilation of the full phase diagram delineating the control-sensitive area for all involved parameters is outside the scope of this paper. 
- Case 6: The phenomenon of synchronization was observed only with the EM field directed normally to the slip surface. When the EM field was applied to the vertical sides of the slipping block, that is, roughly parallel to the slip plane, we were not able to observe the effect of synchronization.

- Case 7: The dominant frequencies, filling individual AE bursts, are in the range of $10-20 \mathrm{kHz}$, no matter whether the experiment is carried out with or without the superimposed mechanical/EM periodic excitation. These frequencies are much larger than the average frequency of slip events and should be related to the slip surface characteristics (Fig. 5d).

- Case 8: Moistening of the slip surface by damp blotting paper does not affect the "mechanical" synchronization, but it practically kills the "electromagnetic" synchronization effect in our conditions that is in the "open" system. "Open" here implies that the gap between the samples is not sealed and has good contact with the atmosphere.

\section{Discussion}

It is well known that the slider-spring system displays stickslip behavior described by nonlinear equations (Dietrich, 1979; Ruina, 1983; Rice and Gu, 1983; Sobolev et al., 1993):

$\tau=\sigma_{n}\left[\mu_{0}+\Theta+A \ln \left(V_{d} / V_{c}\right)\right]$

$\dot{\Theta}=\left(-V_{d} / d_{c}\right)\left[\Theta+B \ln \left(V_{d} / V_{c}\right)\right]$,

where $\tau$ is the friction stress, $\sigma_{n}$ is the normal stress, $\Theta$ is the surface state parameter, $\mu_{0}$ is a nominal (constant) value of friction, $d_{c}$ is the dimension of asperity, $V_{d}$ is the slip speed, $V_{0}$ is the initial value of $V_{d}$, and $A$ and $B$ are constants. Both theoretical solutions and experiments demonstrate the possibility of very different behaviors of the system depending on the conditions of the test. It has also been shown that at the critical value of spring stiffness the friction stress may undergo oscillations that are close to periodic.

Theoretical analysis of a single slider model with a two state-variable rate and state dependent friction shows that such a system is deterministically chaotic and that the road to chaos follows the universal period doubling route (Becker, 2000).

On the other hand, it has been shown (Ott et al., 1990; Bocaletti et al., 2000) that it is possible to control the behavior of chaotic systems using a very small feedback impact. The problem is that the attractor of a chaotic system contains an infinite number of unstable periodic orbits. Given such an attractor, one can choose some of the low-period orbits (or steady states) embedded in the attractor and use a feedback perturbation of an accessible parameter $P$ of the system, in order to stabilize the chosen orbit and thus improve the performance of the system; for example, converting the chaotic behavior in a periodic process. The extreme sensitivity of chaotic systems to the external impact allows for control of the dynamic state of the physical object by using a very small perturbation. It is important that for the parametric control it is not necessary to know the mathematical equations describing the dynamics of the system; it is enough to have experimental time series, measured for some variable $z(t)$, defining a vector $\boldsymbol{X}$, by using the delay coordinate method (Takens,1981). The experimental control of chaos was first successfully realized by Ditto et al. (1990) using parametrically driven magneto-elastic ribbons. This was followed by many others applying chaos to mechanical, electronic, biological and chemical systems (see Ott et al., 1994).

In natural systems, due to their heterogeneity and strong interactions between components, the situation is more complicated than in simple theoretical models. However, that does not exclude the possibility of the appearance of nonlinear structures even in these complex systems. There is clear evidence that not only low dimensional chaos is prone to control, but also high-dimensional chaotic processes, such as the driven double pendulum, can be successfully controlled (Bocaletti et al., 2000).

There are different stabilization and control techniques for chaotic dynamics. The main strategies are the closed loop or feedback methods that has been described above (Ditto et al., 1990), and the open loop or nonfeedback methods (Bocaletti et al., 2000). The second class of methods is based on the application of external perturbation, in order to affect the evolution of the system. Both periodic and stochastic excitations produce changes in the nonlinear behavior of the system; this makes it possible to drive the system to stabilization at some periodic orbit just by varying parameters of the external impact. The drawback of this largely empirical approach is that unlike the targeted feedback method, it is not goal oriented, i.e. the operator cannot predict theoretically the final periodic state without preliminary learning the system's response to variations in parameter space.

As we did not know in detail the behavior of the real slider system (we did not even know whether the dynamics were chaotic or not), we used the nonfeedback method of stabilization. Namely, we imposed periodicity on the continuous component of field $A_{0}$ by the application of a control signal of some frequency $\omega$ and amplitude $\delta A$. The summary signal then is:

$A=A_{0}+\delta A \cos (\omega t)$.

Scanning the frequency and amplitude space at the fixed drag velocity and spring stiffness allows for the detection of suitable parameters that lead to the mode-locking or stabilization of some unstable orbit embedded in the nonlinear structure.

An alternative mathematical formalism for the explanation of the control phenomenon is provided by synchronization theory (Blekhman, 1971; Lursmanashvili et al., 1987), developed in radio engineering. The crux of this approach is using the existence of some critical parameter in the system that causes its relaxation. Then a small periodic impact can synchronize the relaxation of the whole system with the period of impact, if some force regularly drives the system close 


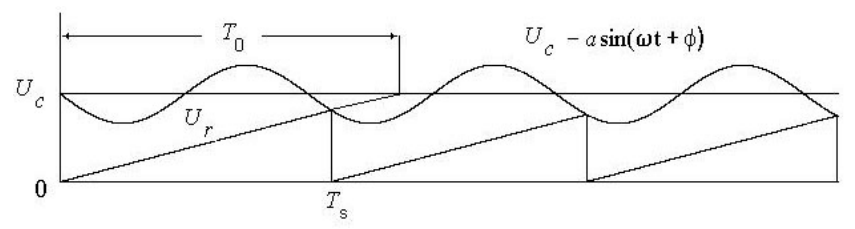

Fig. 6. The simplest synchronization model of the relaxation process. $U_{c}$ is the critical value of intensity $U$, when the rising intensity $U_{r}$ drops to its initial value (here $U_{r}=0$ ); the natural relaxation time of the process is $T_{0}$. If the sinusoidal impact of the intensity $a \sin (\omega t+\varphi)$ is superimposed on $U_{r}$, the condition of the instant drop of intensity $U_{r}$ became: $U_{r}=U_{c}-a \sin (\omega t+\varphi)$ and the period of synchronized oscillations became $T_{S}$. In Fig. 6 the amplitude of superimposed sinusoid is exaggerated.

to the critical state. Let us consider some relaxation process in which the intensity $U_{r}$ (it can be related to voltage, stress, etc.) builds up slowly to some critical value $U_{c}$; when $U_{r}=U_{c}$, the intensity drops instantly to some initial value. Then the application of the synchronizing pulses of the relatively small amplitude $U_{s}$ and of very short duration may impose coherency of these drops with the timing of pulses, as now the condition of criticality is

$U_{r}+U_{s}=U_{c}$

or, in the case of sinusoidal impact,

$U_{r}+a \sin (\omega t+\varphi)=U_{c}$,

where $\omega, a$ and $\varphi$ are, accordingly, the angular frequency, phase and amplitude of the periodic impact. This means that the intensity drops occur (Fig. 6) when the increasing value of $U_{r}$ is equal to $U_{c}-a \sin (\omega t+\varphi)$. It has been shown (Blekhman, 1971) that synchronization may appear at even weak coupling between objects that have significantly different characteristic frequencies, implying nonlinear interaction of objects.

What is the physical mechanism leading to synchronization? In the case of mechanical excitation, synchronization is connected with mechanical triggering of microslips in the system that is close to the critical state, and thus reveals sensitive dependence on (small) external perturbation. The triggering occurs once per period.

As we noted earlier, under EM excitation microslips occur twice per period. We assume that EM synchronization is connected with the polarization of surfaces of fixed and sliding samples. As the polarization forces arise at both polarities of the applied periodic field, it seems reasonable that the synchronization follows each reversal of the EM field.

The role of the EM field direction can be explained as follows. According to our earlier experiments, with the EM impact applied to the system of inclined support-sliding block, the slip is initiated by a strong EM pulse with some finite probability at the slope, less than critical only when the EM field is directed parallel to the slip surface. When the field is normal to the slip plane, the upper block remains stable even at the slope of support, larger than critical; this means that in the latter case the EM field "glues" the surfaces and hampers the slip (Chelidze et al., 2002).

In principle, the external electrical field can affect the inter-surface adhesion (friction) forces, changing surface force balances and thus initiating/blocking the slip of the body. Taking this effect into account, we can rewrite the well-known expression for friction force $F_{f}$ as follows:

$F_{f}=\mu\left(F_{n}+F_{p}\right)$,

where $\mu$ is the friction coefficient and $F_{p}$ is the increment (decrement) of the normal component of force $F_{n}$ due to the application of the EM field.

It is well known that the application of the EM field to the dielectric invokes some forces acting upon molecules of the body. Their resultant is called the ponderomotive force $F_{p}$ and it affects the whole sample. The force is proportional to the gradient of the field intensity squared and it carries away the sample in the direction of the largest intensity. Assuming that the sample of the dielectric constant $\epsilon$ is surrounded by an immobile dielectric medium in the CGS system, then the tension tensor $\mathbf{T}_{n}$ operating on the element of the dielectric's surface in the EM field of intensity $E$ is (Tamm, 1966):

$\mathbf{T}_{n p}= \pm \frac{\epsilon \pm \frac{\partial \epsilon}{\partial \delta} \delta}{8 \pi} E^{2} \boldsymbol{n}$.

Here, the $(+)$ sign corresponds to the case when the field $\boldsymbol{E}$ is parallel to $\boldsymbol{n}$ the external normal to the considered surface element. The $(-)$ sign corresponds to the case when the field $\boldsymbol{E}$ is normal to $\boldsymbol{n}$. We define the former case as the first mode of excitation and the latter as the second mode of excitation.

For a physical interpretation of Eq. (7) we can imagine that the elastic strings are stretched along field lines (Tamm, 1966). In our case they pull together the surfaces of sliding and supporting samples when the sign in Eq. (7) is (+) and build the side thrust on each other when the sign is $(-)$.

Equations (6) and (7) can be simplified if the dielectric increment due to the striction force is negligible, i.e. $(\partial \epsilon / \partial \delta \rightarrow 0)$. Introducing the area of the dielectric surface $S$ and taking into account the above assumption, the ponderomotive force is:

$\boldsymbol{F}_{p}= \pm \frac{\epsilon}{8 \pi} E^{2} \boldsymbol{n}= \pm \frac{\epsilon S}{8 \pi}\left(\frac{\Delta V}{d}\right)^{2} \boldsymbol{n}$,

where $\Delta V$ is the applied voltage and $d$ is the distance between the electrodes. The sign depends on the mutual orientation of dielectric surface and the electrical field.

In order to assess the forces acting in the narrow gap between the slipping and supporting samples, it is necessary to consider the gradient $(\Delta V / d)$ in the gap between the samples where its value is maximum due to the high resistivity of air. Since the opening of the gap varies due to asperities, we can introduce some effective values of opening $d_{\text {eff }}$ and

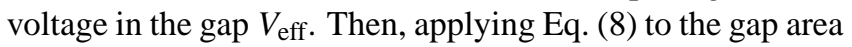
we obtain the ponderomotive force $v e c F_{p i}$ acting on the gap surfaces:

$\boldsymbol{F}_{p i}= \pm \frac{\epsilon_{\mathrm{eff}} S}{8 \pi}\left(\frac{\Delta V_{\mathrm{eff}}}{d_{\mathrm{eff}}}\right)^{2} \boldsymbol{n}$, 
where $V_{\text {eff }} / d_{\text {eff }}$ is the effective voltage gradient in the gap. The effective dielectric constant of the gap, $\epsilon_{\mathrm{eff}}$, is between values of $\epsilon$ for the air and the sample, $1<\epsilon<5$.

Thus, Eq. (6) can be rewritten, taking into account Eq. (9) as:

$F_{f}=\mu\left(F_{n}+F_{p i}\right)$

which is similar to the expression for the friction force with the pore pressure term (Sibson, 1994).

Assuming $\epsilon_{\text {eff }}=2.5$ and $S=100 \mathrm{~cm}^{2}$, we substitute into Eq. (9) the gradient $\left(\Delta V_{\text {eff }} / d_{\text {eff }}\right)=0.07 \mathrm{~V} / \mathrm{cm}$. We do this in order to obtain the experimental values of the EM (ponderomotive) force that initiate slip of the basalt sample on the inclined basalt support at a slope slightly less than critical, namely $F_{p} \approx 0.2 \mathrm{~N}$ (Chelidze et al., 2002). Initiating slip at the same slope, using a mechanical pull, applied through a dynamometer, the calibration of the EM slip-initiating force is performed.

The above value of $F_{p i}$ is of the same order of magnitude of ponderomotive force that promotes the slip in the first mode and hampers it in the second mode, according to the expression in Eq. (10) for the accepted set of parameters. The same order was obtained for the mechanical equivalent of the EM force in the present experiments: control of slip appears at the application of AC voltage, (approximately) $300 \mathrm{~V}$ at EM excitation or at the application of mechanical periodic force (approximately) of amplitude $0.1-0.5 \mathrm{~N}$. The low frequency of periodic excitations in our experiments justifies the application of the above static treatment and in particular, using Eq. (9) for the physical interpretation of the EM control of slip.

In general, the elastic systems manifest unstable frictional slip under the condition of reduction of the friction force during some phase of sliding; this property is called slipweakening. Our guess is that in our experiments the unstable sliding regime (slip-weakening) is imposed by the pulling force, but the synchronization of microslips by periodic electric force, applied in the first mode, gives the system the (periodic) negative feedback, causing transient intervals of slip-hardening. Overcoming these periodic "electromagnetic asperities" by the mechanical pull causes synchronous microslips (acoustic bursts). The EM field, applied in the second mode, i.e. parallel to the slip surface, does not cause sliphardening and consequently, results in synchronization.

As mentioned earlier, making the slip surface damp with blotting paper did not affect the "mechanical" synchronization, but it strongly reduced or killed the electromagnetic synchronization effect in our conditions, when the slip surface had a free access to the atmosphere. This can be explained in terms of Eq. (9) as a result of a decrease in voltage gradient in the gap between fixed and slipping samples due to the high conductivity of the filling fluid. It cannot be excluded that in confined systems, i.e. when the gap is sealed and pore pressure on the slip surface can be controlled, electrokinetic and other processes in the gap can provoke the EM synchronization effect.
The results obtained confirm in principle the possibility of controlling the regime of the natural seismicity at least in the temporal domain by relatively weak artificial (strong EM pulses) or natural (magnetic storms, tides) excitations. For example, there are reliable experimental data on the strong synchronization of microtremors in volcanic areas with Earth's tides (Custodio, 2002). It is also shown by statistical methods that magnetic storms with sudden commencement and strong artificial EM discharges may affect the seismic regime (Zakrzhevskaya and Sobolev, 2002; Chelidze and Matcharashvili, 2003).

\section{Conclusions}

Experiments on the spring-slider system (fixed and sliding basalt samples), subjected to a constant pull and superimposed with weak mechanical or EM periodic forces in a dry environment, show that at definite conditions the system manifests synchronization of microslip events with weak excitation. The regimes of slip vary from the perfect synchronization of slip events (acoustic emission) with the perturbing periodic mechanical or EM impact, to the complete desynchronization of microslip events and perturbations. The phenomenon can be explained in terms of nonlinear dynamics and synchronization theory.

We conclude that our laboratory experiments give a sound principal basis for the interpretation of the field data on the control of seismic regime by relatively weak natural or artificial perturbations; of course, in the Earth's crust, the detailed physical mechanisms of coupling between tectonic forces and superimposed perturbations may be different.

Acknowledgements. This work was supported by the INTAS-open grants \# 64-99 and INTAS \# 01-0748.

\section{References}

Becker, T. W.: Deterministic Chaos in the Two State-variable Friction Sliders and the Effect of Elastic Interactions, in: Geocomplexity and the Physics of Earthquakes, edited by Rundle, J., Turcotte, D., and Klein, W., AGU, Washington, DC, 5-26, 2000.

Blekhman, M. M.: Synchronization of dynamical systems, Moscow, Nauka Publ. House (in Russian), 1971.

Bocaletti, S., Grebogi, C., Lay Y.-C.: The control of chaos; theory and applications, Physics Reports, 329, 103-197, 2000.

Bocaletti, S., Grebogi, C., Lay, Y.-C., Manchini, H., Maza. D.: The Control of Chaos: Theory and Applications, Physics Reports, 329, 103-197, 2000.

Chelidze, T. and Matcharashvili, T.: Electromagnetic Control of Earthquake Dynamics? (Computers \& Geosciences), 29, 587593, 2003.

Chelidze, T., Varamashvili, N., Devidze, M., Tchelidze, Z., Chikhladze, V.: Laboratory Study of Electromagnetic Initiation of Slip, Annals of Geophysics, 45, 587-599, 2002.

Custodio, S., Fonseca, J., Faria, B., d'Oreye, N.: Tidal modulation of volcanic tremor in Fogo Island, Cape Verde, Book of Abstracts, European Seismological Commission XXVIII Assembly, Genova, 236, 2002. 
Dieterich, J. H.: Modelling of rock friction 1., J. Geophys. Res., 84, 2162-2168, 1979.

Ditto, W. L., Rauseo, S. N., and Spano, M. L.: Experimental control of chaos, Phys. Rev. Lett., 65, 3211-3214, 1990.

Lursmanashvili, O., Gakhokidze, L., Nikoladze, I., and Ruda, L.: The Results of a study of the repetition spectrum of earthquakes in the Caucasus, Bulletin of the Georgian Academy of Sciences, 126, 73-76, 1987.

Ott, E., Grebogi, C., and Yorke, J. A.: Controlling chaos, Phys. Rev. Lett., 64, 1196-1199, 1990.

Ott, E., Sauer, T., and Yorke, J. A.: Coping with Chaos, John Wiley\&Sons, N.Y., 1994.

Rice, J. and Gu, Y.: Earthquake aftereffects and triggering seismic phenomena, P. A. Geoph., 121, 443-475, 1983.

Ruina, A.: Slip instability and state variable friction law, J. Geophys. Res., 88, 10359-10370, 1983.

Sibson, R.: Crustal stress, faulting and fluid flow, in: Geofluids: Origin, Migration and Evolution of Fluids in Sedimentary
Basins, edited by Parnell, J., The Geological Society, London, 69-84, 1994.

Sobolev, G., Spetzler, H., Koltsov, A., and Chelidze, T.: An experimental study of triggered stick-slip, P. A. Geoph., 140, 79-94, 1993.

Tamm, I.: Fundamentals of theory of electricity, "Nauka" Publ. House, Moscow, (in Russian), 1966.

Takens, F.: Detecting strange attractors in turbulence, in: Dynamical Systems and bifurcations, edited by Rand, D. and Young, L., Springer Lectures in Mathematics, 898, 366-381, 1981.

Tarasov, N., Tarasova, H., Avagimov A., and Zeigarnik, V.: The effect of high-power electromagnetic pulses on the seismicity of Central Asia and Kazakhstan, Volcanology and Seismology (Moscow), (in Russian), 4-5, 152-160, 1999.

Zakrzhevskaya, N. and Sobolev, G.: On the Seismicity Effect of Magnetic Storms, Izvestia, Physics of Solid Earth, 38, 249-261, 2002. 